\title{
Gammarus: Important Taxon in Freshwater and Marine Changing Environments
}

\author{
Almut Gerhardt, ${ }^{1}$ Michelle Bloor, ${ }^{2}$ and Chris Lloyd Mills ${ }^{3}$ \\ ${ }^{1}$ LimCo International GmbH, Technologiezentrum Konstanz, Blarerstrße 56, 78462 Konstanz, Germany \\ ${ }^{2}$ School of Earth and Environmental Science, University of Portsmouth, Burnaby Building, Burnaby Road, \\ Portsmouth PO1 3QL, UK \\ ${ }^{3}$ School of Science and Technology, Nottingham Trent University, Clifton Lane, Nottingham NG11 8NS, UK \\ Correspondence should be addressed to Almut Gerhardt, almutg@web.de
}

Received 19 June 2011; Accepted 19 June 2011

Copyright ( $) 2011$ Almut Gerhardt et al. This is an open access article distributed under the Creative Commons Attribution License, which permits unrestricted use, distribution, and reproduction in any medium, provided the original work is properly cited.

Gammarus spp. consist of more than 100 freshwater, brackish, and marine species in the Northern hemisphere. They represent important keystone species in aquatic ecosystems and are often present in high abundance. As shredders and detritus feeders, they contribute to the detritus cycle and the microbial loop. Gammarids are also carnivorous, feeding on small invertebrates and carrion. Due to their widespread distribution, significance in the food web, and sensitivity to a wide range of pollutants, they are important bioindicators for water quality assessment. Gammarus spp. are ecologically highly successful due to the following characteristics: wide trophic repertoire and foraging plasticity, migration ability and tendency to drift, which allows them to easily invade and colonize ecosystems, high reproductive capacity with several broods per female per year, and a high number of offspring and relatively longevity (1-2 yrs).

Gammarus spp. and their American relative Hyalella azteca are standard test species in ecotoxicity testing in the USA and UK. A new OECD test guideline is currently being prepared for gammarids, which will consist of a variety of in situ and ex situ ecotoxicological studies based on different measurement parameters.

The evolution of gammarids is particularly interesting: gammarids contain several Ponto-Caspian and Atlantic invasive species, which have spread throughout Europe. Some species are currently being divided into several geographical forms. Although the genome of Gammarus pulex has yet to be sequenced, 12345 expressed sequence tags are known, which might be the basis for future innovative knowledge in taxonomy and toxicogenomics.

Despite the taxon's importance in different fields of biology (e.g., ecology, evolution, ecotoxicology, taxonomy, biogeography), its application (as test species and bioindicators) in scientific research tends to consider isolated questions. The aim of this special issue on Gammarus spp. is to highlight the importance of this taxon and facilitate interdisciplinary research. The focus of this issue is to present articles that showcase different facets of gammarid research, including methods that demonstrate direct practical approaches. In order to reach a wide spectrum of readers from different disciplines and geographical regions, we chose this journal with global open access.

Gammarids are known to be important shredders in the aquatic detritus cycle and the microbial loop. The most recent research within the field of gammarid-microbial interactions is summarized and discussed in the first article by D. Nelson. Gammarids prefer so-called conditioned leaves as food, that is, leaves colonized by a microbial biofilm. The role of hyphomycete fungi as a source of secondary metabolites is stressed in the paper. However, although the effects of leaf type and microbial colonization on Gammarus spp. feeding activity, growth, and survival has been studied, the effects of Gammarus spp. on shaping the microbial community still remain unknown.

Understanding dietary requirements of gammarids is essential knowledge to describe feeding schemes for a 
successful laboratory culture. M. Bloor tests unconditioned and conditioned (either naturally conditioned or artificially conditioned) alder leaves as a food source for Gammarus pulex and Asellus aquaticus and concludes that both species prefer naturally conditioned leaves compared to artificial conditioning. M. Bloor provides a simple method to prepare alder leaves for laboratory culturing of both species. Successful breeding is an essential requisite for a standard test species in ecotoxicological test procedures.

A. Gerhardt presents a simple low-cost test system with Gammarus spp. studying survival, feeding, and behaviour as well as biomarkers, to be used both in and ex situ. This test protocol does not need sophisticated laboratory equipment and hence can be applied worldwide. Compared to the standard test species Daphnia magna, gammarids tend to be as sensitive towards toxicants and are more ecologically relevant test species. We hope to increase the application of gammarids in aquatic ecotoxicology by providing simple methods for breeding, feeding, and test systems.

Whilst gammarids can be very sensitive towards pesticides, they are also susceptible to natural defence substances released by aquatic plants. M. J. Dixon and P. J. Shaw show that both pulses of wash water from a watercress farm and isolated phenethyl isothiocyanate (PEITC) can influence the reproductive behaviour of Gammarus pulex. Short-term pulses lead to the interruption of precopula pairs, with recovery occurring in clean water. Such a transient behavioural parameter has the potential to be used as an early warning indicator of pulsed pollution stress.

A sound basis for every ecological and ecotoxicological study is species determination. In gammarids several cryptic species have been found based on genetic methods. However, the proof of mechanisms in speciation such as geographical and/or ecological isolation is still lacking. Do genetic differences suffice to describe (sub)species, or do we need a combination of genetic, morphological, and ecological methods? Haug et al. present the most recent methods for imaging gammarids from whole animals to tiny structures using both high- and low-cost techniques. Modern imaging methods in combination with sophisticated software are very powerful tools which facilitate taxonomic determination. In this paper, different techniques are presented, from simple light microscopy with staining techniques, autofluorescence, and polarized light applications to focused ion beam scanning electron microscopy, confocal laser microscopy, and microtomography.

Although most gammarids represent freshwater Gammarus species, brackish and marine gammarids have recently been studied in more detail. L. Delgado et al. show that both adult and juvenile Gammarus aequicauda have a wide salinity tolerance range and that juvenile growth rate is only affected towards the extremes of the salinity tolerance range. Ecological plasticity is a prerequisite to spread to different habitats and geographical locations. Gammarids are highly successful in this aspect.

A. Mirzajani et al. describe selection of gammarid species for potential use as a natural food source for warm water fish farming in Iran. In this interesting approach the reproductive traits of 7 amphipod species have been studied with respect to their breeding periods and reproductive output. They identified 4 species that might be suitable for warm water fish aquaculture.
Almut Gerhardt Michelle Bloor Chris Lloyd Mills 

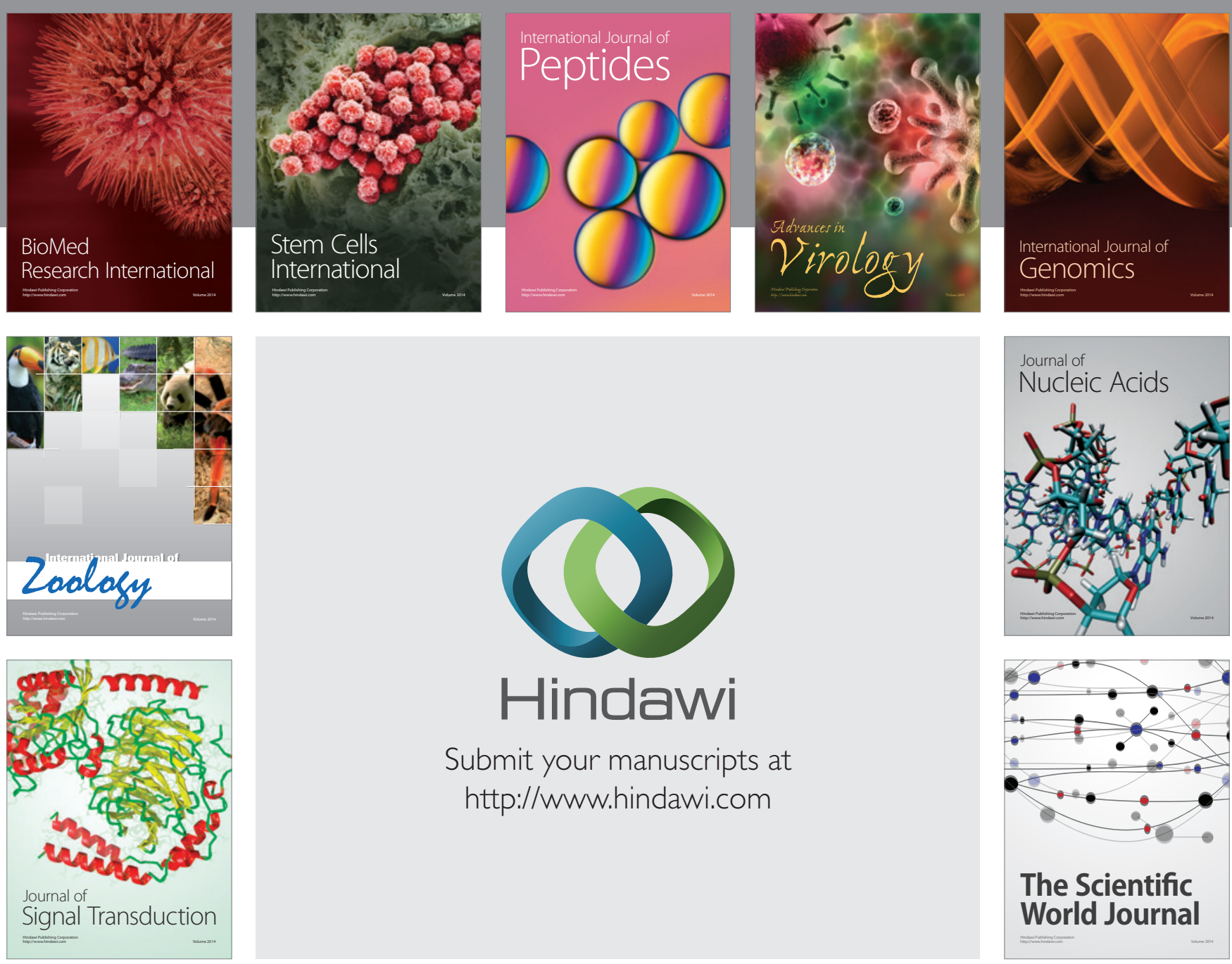

Submit your manuscripts at

http://www.hindawi.com
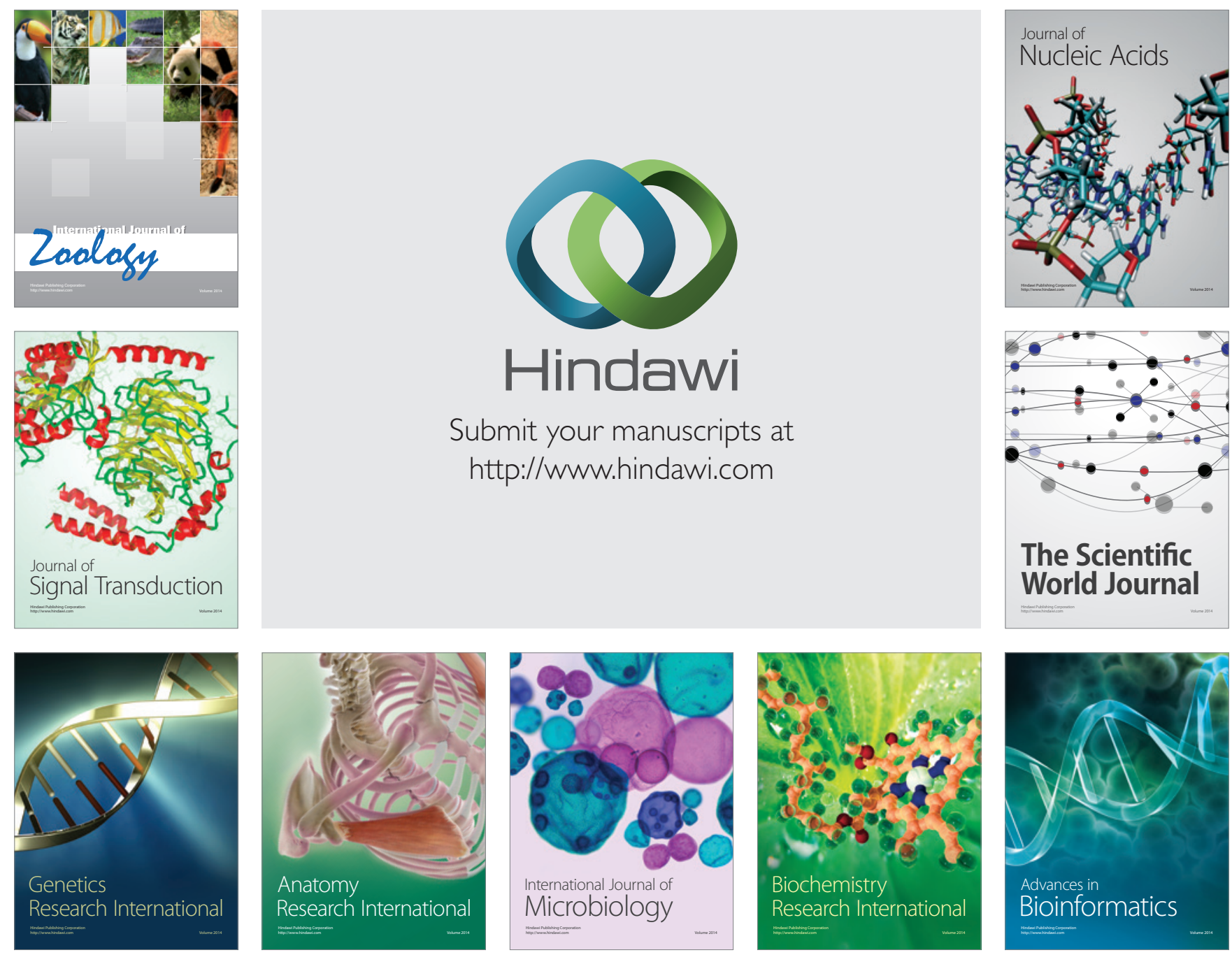

The Scientific World Journal
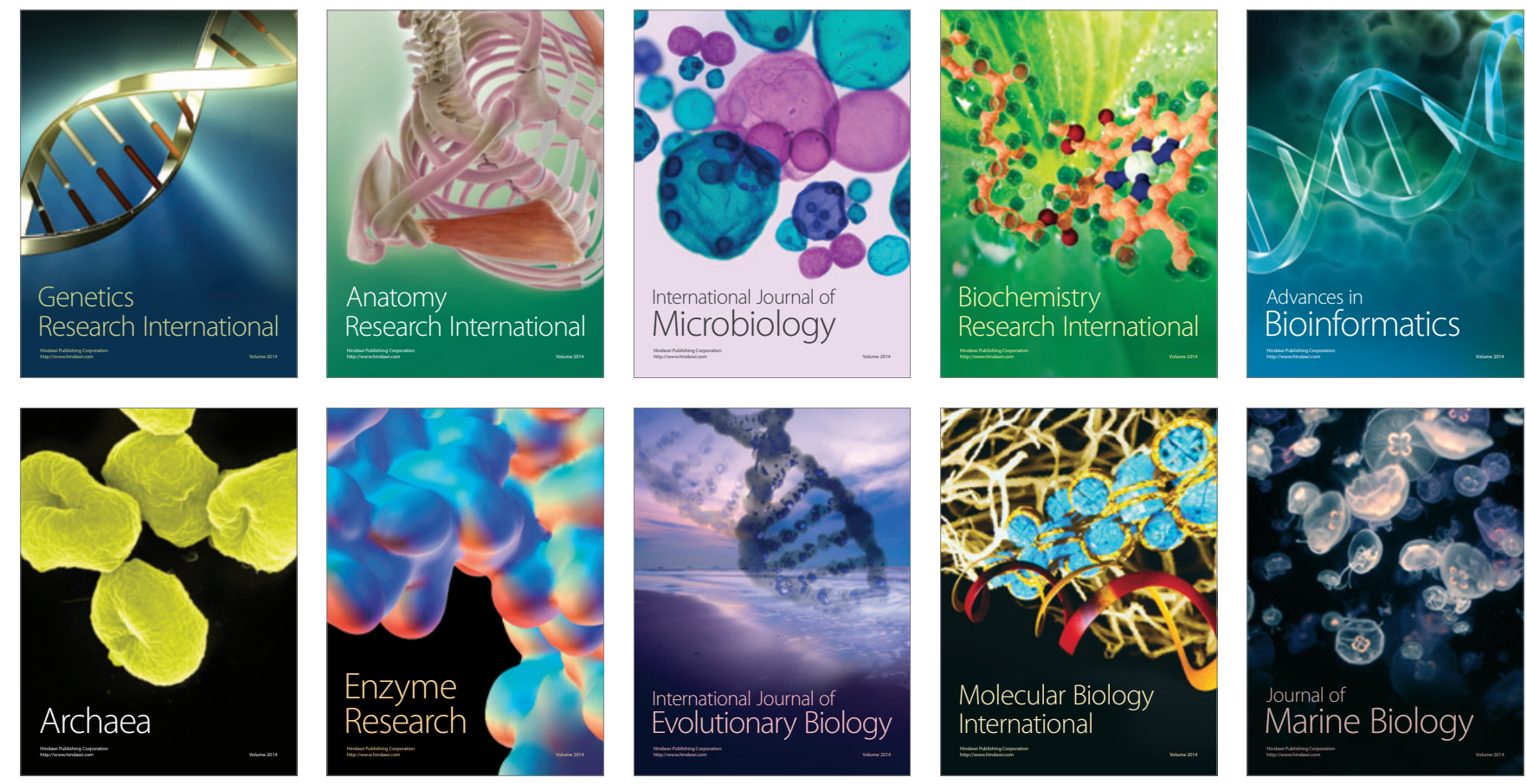